\title{
Large normal subgroup growth and large characteristic subgroup growth
}

\author{
Yiftach Barnea and Jan-Christoph Schlage-Puchta
}

Communicated by Miklós Abért

\begin{abstract}
The fastest normal subgroup growth type of a finitely generated group is $n^{\log n}$. Very little is known about groups with this type of growth. In particular, the following is a long standing problem: Let $\Gamma$ be a group and $\Delta$ a subgroup of finite index. Suppose $\Delta$ has normal subgroup growth of type $n^{\log n}$. Does $\Gamma$ have normal subgroup growth of type $n^{\log n}$ ? We give a positive answer in some cases, generalizing a result of Müller and the second author and a result of Gerdau. For instance, suppose $G$ is a profinite group and $H$ an open subgroup of $G$. We show that if $H$ is a generalized Golod-Shafarevich group, then $G$ has normal subgroup growth of type $n^{\log n}$. We also use our methods to show that one can find a group with characteristic subgroup growth of type $n^{\log n}$.
\end{abstract}

\section{Introduction and results}

For a group $\Gamma$, let $s_{n}^{\triangleleft}(\Gamma)$ be the number of normal subgroups of $\Gamma$ of index at most $n$. Very little is known about the possible asymptotic behavior of this sequence; see [7, Chapter 2 and Section 9.4] for background. Lubotzky [6] showed that, for any finitely generated group, we have $s_{n}^{\triangleleft}(\Gamma) \ll n^{c \Omega(n)}$ for some constant $c$, where $\Omega(n)$ denotes the number of prime divisors of $n$ counted with multiplicity, and Mann [9] showed that, for a non-abelian free group, we have $s_{n}^{\triangleleft}(\Gamma)>n^{c \log n}$ for some $c>0$ and infinitely many $n$. Comparing these results, we find that the normal subgroup growth of a non-abelian free group is of type $n^{\log n}$. We say that a function $f(n)$ is of type $g(n)$ if there are constants $c_{1}, c_{2}$ such that $\log f(n) \leq c_{1} \log g(n)$ for all $n$, and there are infinitely many $n$ such that $\log f(n)>c_{2} \log g(n)$.

Our first theorem, Theorem 2, is quite technical. So, to motivate the reader, we start with Corollary 1 which might have more general interest. It concerns characteristic subgroup growth. In general, the characteristic subgroup growth of a group is even more mysterious than the normal subgroup growth; in particular, determining the characteristic growth of a free non-abelian group appears to be quite difficult. However, the following is a simple consequence of Theorem 2.

Corollary 1. Let $\Gamma$ be a virtually non-abelian free group with trivial center, and suppose $\Gamma$ has a finite outer automorphism group. Then $\Gamma$ has characteristic sub- 
group growth of type $n^{\log n}$. In particular, if $\Gamma=A * B$ is the free product of two non-trivial finite groups such that at least one has order greater than 2 , then $\Gamma$ has characteristic subgroup growth of type $n^{\log n}$.

We return now to consider normal subgroup growth. One of the basic problems concerning the normal subgroup growth is the question of whether we can compare the normal subgroup growth of a group and of a subgroup of finite index. If $\Delta<\Gamma$ is a subgroup of finite index, we can intersect normal subgroups of $\Gamma$ with $\Delta$, that is, if $\Gamma$ has many normal subgroups, so has $\Delta$. More precisely, a slight variation of the proof of [7, Proposition 1.3.2 (ii)] gives us $s_{n}^{\triangleleft}(\Gamma) \leq s_{n}^{\triangleleft}(\Delta) n^{(\Gamma: \Delta)}$. Therefore, unless we are aiming at results of high precision, the difficult problem is to decide whether a finite index subgroup of a group $\Gamma$ can have substantially more normal subgroups than $\Gamma$ itself. Lubotzky and Segal [7, Problem 4(a)] ask the following.

Problem 1. Let $\Gamma$ be a group and $\Delta$ a subgroup of finite index. Suppose $\Gamma$ has polynomial normal subgroup growth, that is, type $n$. Does $\Delta$ have polynomial normal subgroup growth?

The fundamental problem we consider is a slight variation on it.

Problem 2. Let $\Gamma$ be a group and $\Delta$ a subgroup of finite index. Suppose $\Delta$ has normal subgroup growth of type $n^{\log n}$. Does $\Gamma$ has normal subgroup growth of type $n^{\log n}$ ?

This basic question seems to be quite hard, and unfortunately we are only able to answer it in special cases. In [10, Theorem 1], Müller and the second author claimed the following theorem. If $\Gamma$ is a finitely generated group and $\Delta$ is a normal subgroup of finite index such that $\Delta$ maps onto a group $G$ such that the pro- $p$ completion of $G$ is a non-abelian free pro- $p$ group for some prime $p$ and $p \nmid(\Gamma: \Delta)$, then $\Gamma$ has normal subgroup growth of type $n^{\log n}$. The proof used Mann's construction of large elementary abelian sections and representation theory to study the action of $\Gamma / \Delta$ on these sections. Gerdau [5] claimed that the condition $p \nmid|\Gamma / \Delta|$ is not necessary. In his proof, Gerdau replaced ordinary representation theory by modular representation theory. Notice that, actually, there is no need to require $\Delta$ to be normal. However, a careful examination of both proofs shows that weaker results are proven, namely, if $\Gamma$ is a finitely generated group and $\Delta$ is a normal subgroup of finite index such that the pro- $p$ completion of $\Delta$ is a non-abelian free pro- $p$ group, then $\Gamma$ has normal subgroup growth of type $n^{\log n}$. We do not know whether the original statements are true. The problem in the proofs is that a group that contains a subgroup of finite index which projects onto a free group needs not project onto a virtually free group. An ex- 
ample of such group is the semi-direct product $C_{2} \ltimes\left(F_{2} \times F_{2}\right)$, where $C_{2}$ acts by interchanging the factors of the direct product. This has a subgroup of order 2 , that projects onto $F_{2}$, but does not project onto any virtually (non-abelian free) group. However, it is still true that all virtually (non-abelian free) groups have normal subgroup growth of type $n^{\log n}$.

In this note, we give a couple of far more general results. Theorem 2 below appears rather technical; however, in concrete cases, the conditions of the theorem are easy to establish. We write $d(\Gamma)$ for the minimal number of generators of $\Gamma$, and if $\Delta$ is a normal subgroup of $\Gamma$, we write $d_{\Gamma}(\Delta)$ for the minimal number of generators of $\Delta$ as a normal subgroup of $\Gamma$; in the case of topological groups, we take topological generators. We call a group a $C M E A$ group if it is a central extension of an elementary abelian $p$-group by an elementary abelian $p$-group. We write $\operatorname{rk}_{c m} G$ for the $C M E A$ rank of a pro- $p$ group $G$, that is, the logarithm to base $p$ of the maximal order of a CMEA image of $G$.

Theorem 2. Let $\Gamma$ be a d-generated group, $\Delta$ a normal subgroup of finite index in $\Gamma$ and $p$ a prime number.

(1) Let $H$ be the pro-p completion of $\Delta$, and let $\Psi$ be the pre-image of $\Phi(H)$ under the canonical map $\Delta \rightarrow H$. Then $d_{\Gamma}(\Psi) \geq \frac{\mathrm{rk}_{c m} H}{(\Gamma: \Delta)}-d$.

(2) Let $c>0$ be a real number. Suppose $\Delta_{i}$ is an infinite sequence of normal subgroups of $\Gamma$ contained in $\Delta$ and of index $p$-power in $\Delta$. If, for all $i$, we have $\mathrm{rk}_{c m} \Delta_{i}>c\left(\Gamma: \Delta_{i}\right)^{2}$, then $\Gamma$ has normal subgroup growth of type $n^{\log n}$. In fact, the number of subgroups of $\Delta$, which are normal in $\Gamma$, has growth type $n^{\log n}$.

Let us see that Theorem 2 implies Gerdau's result (as proved rather than as claimed). Let $\Gamma$ and $\Delta$ be as in Gerdau's result and $H$ the pro- $p$ completion of $\Delta$, by our assumption $H$ is a free non-abelian pro- $p$ group, and let $r \geq 2$ be its number of generators. Write $\widehat{\Gamma}$ and $\widehat{\Delta}$ for the profinite completion of $\bar{\Gamma}$ and $\Delta$, respectively. Then $\widehat{\Gamma}$ contains $\widehat{\Delta}$ because $\Delta$ is of finite index in $\Gamma$. Write $K$ for the kernel of the map from $\widehat{\Delta}$ to $H$ and, trivially, $\widehat{\Delta} / K \cong H$. Clearly, $H$ is the pro- $p$ completion of $\widehat{\Delta}$, so $K$ is a characteristic subgroup of $\widehat{\Delta}$ and therefore normal in $\widehat{\Gamma}$. Then $\Gamma$ maps densely into $G=\widehat{\Gamma} / K$, and $\widehat{\Delta} / K \cong H$ is contained in $G$.

Let $N$ be a normal subgroup of finite index of $G$ contained in $H$ and $\Omega$ its pre-image in $\Delta$. Since $N$ is a free pro- $p$ group, it is mapped onto the free CMEAgroup

$$
\begin{gathered}
\left\langle x_{i}, y_{i}, z_{i k}\right| 1 \leq k<i \leq d, x_{i}^{p}=y_{i},\left[x_{i}, x_{k}\right]=z_{i k}, \\
\left.y_{i}^{p}=z_{i}^{p}=\left[x_{i}, y_{j}\right]=\left[x_{i}, z_{j k}\right]=1\right\rangle
\end{gathered}
$$

with $d=(H: N)(r-1)+1=(\Delta: \Omega)(r-1)+1$, and hence $\Omega$ is mapped onto 
the same free CMEA-group. In particular, we have $\operatorname{rk}_{c m} \Omega \geq d+\left(\begin{array}{c}d+1 \\ 2\end{array}\right)$. Take

$$
c=\frac{(r-1)}{(\Gamma: \Delta)}>0
$$

which is independent of $N$; then

$$
d=c(\Gamma: \Delta)(\Delta: \Omega)+1=c(\Gamma: \Omega)+1 .
$$

Therefore, $\left(\begin{array}{c}d+1 \\ 2\end{array}\right)>d^{2} / 2>\left(c^{2} / 2\right)(\Gamma: \Omega)^{2}$. It follows from Theorem $2(2)$ that $\Gamma$ has normal subgroup growth of type $n^{\log n}$.

Next we would like to consider virtual Golod-Shafarevich groups. However, to consider non-normal subgroups, we need to be able to change to a normal subgroup of finite index, but subgroups of finite index of Golod-Shafarevich groups are not necessarily Golod-Shafarevich. Thus, we consider the larger class of generalized Golod-Shafarevich groups introduced by Ershov and Jaikin-Zapirain [4]. We postpone the formal definition of a generalized Golod-Shafarevich group to the next section; more detailed treatments can be found in [4] and [3, Chapter 5].

Theorem 3. Let $G$ be a profinite group and $H$ an open subgroup which is a generalized Golod-Shafarevich pro-p group. Then $G$ has normal subgroup growth of type $n^{\log n}$.

The proof of Theorem 3 requires no representation theory; nevertheless, it also implies the result of Gerdau. Indeed, let $\Gamma$ and $\Delta$ be as in Gerdau's result. We take $\widehat{\Gamma}, \widehat{\Delta}, H$ and $K$ as above. By our assumption, $H$ is a non-abelian free pro- $p$ group. As $\Delta$ is normal in $\Gamma$ (we can always assume that by passing to a subgroup of finite index), $\widehat{\Delta}$ is normal in $\widehat{\Gamma}$. Hence, $K$ is normal in $\widehat{\Gamma}$. Let $G=\widehat{\Gamma} / K$, and the result follows since non-abelian free pro- $p$ groups are generalized Golod-Shafarevich groups.

Both theorems imply that groups of virtual positive $p$-deficiency have normal subgroup growth of type $n^{\log n}$. For the notion of $p$-deficiency, we refer the reader to [13]. This is easy to see for Theorem 3 since groups of positive $p$-deficiency are virtually Golod-Shafarevich (see [1, Theorem 5.5]). For Theorem 2, this requires some computation similar to the case of virtually free groups, which we skip here.

Next we give an example, where Theorem 2 is applicable, but Theorem 3 is not.

Proposition 4. Let $G$ be the quotient of the free pro- $p$ group $F$ in $d \geq 2$ generators by $\gamma_{2}\left(F^{\prime}\right)$. Then $G$ has normal growth type $n^{\log n}$.

This result is sharp in view of a result by Segal [12], who showed that metabelian groups have normal growth of type $n^{(\log n)^{1-\delta}}$ for some $\delta>0$. In other words, abelian by abelian groups cannot have large normal growth, while (nilpotent of class 2) by abelian can. 
We do not have an example of a Golod-Shafarevich group which does not satisfy the conditions of Theorem 2, but we believe that such examples should exist. In particular, if $G$ is a Golod-Shafarevich group of subexponential subgroup growth, then Theorem 3 is applicable, but Theorem 2 is not. While it is likely that such groups exist, no examples are known to us.

Finally, we remark that both Theorem 2 and 3 apply to Fuchsian groups of positive hyperbolic volume. Such a group has a normal subgroup of finite index, which is a surface group with at least 4 generators, and both the fact that surface groups map onto large CMEA groups and that the pro- $p$ completion of surface groups is Golod-Shafarevich follow immediately from the definition. This is important as the normal subgroup growth of Fuchsian groups was used in [14] to count isomorphism types of algebraic curves with many automorphisms, and the corrected version of [10] does not suffice for this purpose.

\section{Virtual Golod-Shafarevich groups}

Lemma 5. A pro-p group $G$ has normal growth of type $n^{\log n}$ if and only if there exists some $c>0$ such that, for infinitely many normal subgroups $N$ of finite index, we have $d_{G}(N)>c \log (G: N)$.

Proof. Suppose $N$ is an open normal subgroup satisfying $d_{G}(N)>c \log (G: N)$. Then $N / \Phi_{G}(N)$ is a vector space of dimension greater than $c \log (G: N)$, and therefore, this space contains at least $p^{(c \log (G: N))^{2} / 4}$ subspaces of codimension $d=\left\lfloor\frac{c}{2} \log (G: N)\right\rfloor$. Since every subspace corresponds to a normal subgroup of index $(G: N) p^{d}$, we conclude that $G$ has normal growth of type $n^{\log n}$.

Suppose on the other hand that there exists a function $f(n), f(n)=o(n)$, such that $d_{G}(N) \leq f(n)$ for all $N \triangleleft G$ with $(G: N)=p^{n}$. Then, for each normal subgroup $N$ with $(G: N)=p^{n}$, there exists a sequence

$$
G=N_{0}>N_{1}>N_{2}>\cdots>N_{n}=N
$$

with $N_{i} \triangleleft G$ and $\left(N_{i}: N_{i+1}\right)=p$. If $N_{i}$ is fixed, $N_{i+1}$ can be chosen in $\frac{p^{d} G^{\left(N_{i}\right)-1}}{p-1}$ ways; hence the number of normal subgroups of index $p^{n}$ is at most

$$
p^{\sum_{v \leq n} f(v)}=p^{o\left(n^{2}\right)},
$$

and we conclude that the normal growth of $G$ is not of type $n^{\log n}$.

Lemma 6. Let $G$ be a profinite group, which contains an open normal subgroup $H$ that is a pro- $p$ group. If there exists some $c>0$ such that $H$ contains infinitely many characteristic open subgroups $N$ satisfying $d_{H}(N)>c \log (H: N)$, then $G$ has normal growth $n^{\log n}$ 
Proof. Suppose $N$ is a characteristic subgroup of $H$ with $d_{H}(N)>c \log (H: N)$. Then $N$ is normal in $G$; let $x_{1}, \ldots, x_{d}$ be elements generating $N$ as a normal subgroup of $G$. Let $g_{1}, \ldots, g_{k}$ be representatives of the cosets $G / H$. Then $x_{i}^{g_{j}}$, $1 \leq i \leq d, 1 \leq j \leq k$, generates $N$ as a normal subgroup of $H$; hence we have $(G: H) d \geq d_{H}(N)$. We conclude that

$$
\begin{aligned}
d_{G}(N) & \geq \frac{d_{H}(N)}{(G: H)} \geq \frac{c}{(G: H)} \log (H: N) \\
& =\frac{c}{(G: H)}(\log (G: N)-\log (G: H)) \\
& \geq \frac{c}{2(G: H)} \log (G: N),
\end{aligned}
$$

provided $(G: H)(H: N)=(G: N)>(G: H)^{2}$, that is, $(H: N)>(G: H)$. The latter condition excludes only finitely many $N$, and our claim follows.

Let $F(X)$ be the free pro- $p$ group over the set $X$. Let $\mathbb{F}_{p}\langle\langle X\rangle\rangle$ be the ring of power series in non-commuting variables over $X$. The map $x \mapsto 1+x$ for all $x \in X$ extends to the Magnus map $\mu: F(X) \rightarrow \mathbb{F}_{p}\langle\langle X\rangle\rangle$. Magnus [8] proved that it is injective. To each element $x \in X$, we associate a positive integer $d_{x}$, which we call the degree of $x$. We can extend this degree to a function $D: \mathbb{F}_{p}\langle\langle X\rangle\rangle \rightarrow \mathbb{N}$ by defining the degree of a monomial to be the sum of the degrees of its factors, and the degree of a linear combination of monomials as the maximal degree of one of these monomials. Now define a degree function $d: F(X) \rightarrow \mathbb{N}$ by putting $d(w)=D(\mu(w)-1)$. It is not hard to see that an element in the $n$-dimension subgroup has degree at least $n$. For a set $A \subset F(X)$, define the Hilbert series $H_{d, A}(t)=\sum_{a \in A} t^{d(a)}$.

Let $\langle X \mid R\rangle$ be a presentation with $X$ finite. We say that this presentation is a generalized Golod-Shafarevich presentation if there exists a degree function $d$ and a real number $t_{0} \in(0,1)$ such that $1-H_{d, X}\left(t_{0}\right)+H_{d, R}\left(t_{0}\right)<0$. A pro- $p$ group is called a generalized Golod-Shafarevich group if it has a generalized GolodShafarevich presentation. Note that the usual notion of a Golod-Shafarevich group corresponds to the degree function which assigns to all $x \in X$ the degree 1 . We use the same terminology as in [3]. The following is contained in [3, Theorem 4.3 and Theorem 4.4].

Proposition 7. The following statements hold.

(1) A generalized Golod-Shafarevich group is infinite.

(2) An open subgroup of a generalized Golod-Shafarevich group is again generalized Golod-Shafarevich. 
In [2, appendix], Jaikin-Zapirain showed that a finitely generated generalized Golod-Shafarevich group has subgroup growth of type at least $p^{n^{\beta}}$ for some $\beta>0$. Using the basic idea from his proof, we show the following lemma. For that, we need to recall that, given a pro- $p$ group $G$, the $n$-dimension subgroups of $G$ is

$$
D_{n}(G)=\prod_{i p^{j} \geq n} \gamma_{i}(G)^{p^{j}} .
$$

Lemma 8. Let $H$ be a finitely generated generalized Golod-Shafarevich pro- $p$ group. Then there exists $c>0$ such that $H$ contains infinitely many open characteristic subgroups $N$ satisfying $d_{H}(N)>c \log (H: N)$.

Proof. Pick a presentation $\langle X \mid R\rangle$ of $H$, a degree function $d$ and a real number $t_{0} \in(0,1)$ such that $1-H_{d, X}\left(t_{0}\right)+H_{d, R}\left(t_{0}\right)=-\delta<0$. Our aim is to show that $D_{n}=D_{n}(H)$ needs many generators as normal subgroups of $H$. We have $d_{H}\left(D_{n}\right) \geq \log \left(D_{n}: D_{n+1}\right)$ since $\Phi_{H}\left(D_{n}\right) \leq D_{n+1}$.

Let $g_{1}, \ldots, g_{m}$ be elements of $H$, which generate $D_{n}$ as a normal subgroup. Then there exist elements $r_{1}, \ldots, r_{m} \in F(X)$, where $F(X)$ is the free pro- $p$ group on the set $X$, such that $r_{i}$ maps to $g_{i}$ under the map $F(X) \rightarrow H$ induced by the presentation. The degree of $r_{i}$ is at least $n$, as $r_{i} \in D_{n}$. Put $\widetilde{R}=R \cup\left\{r_{1}, \ldots, r_{m}\right\}$. Then $\langle X \mid \widetilde{R}\rangle=H / D_{n}$ is a finite group; in particular, this group does not satisfy the generalized Golod-Shafarevich inequality. The Hilbert series of $\widetilde{R}$ is

$$
H_{d, R}(t)+\sum_{i=1}^{m} t^{d\left(r_{i}\right)},
$$

and hence

$$
\begin{aligned}
0 & \leq 1-H_{d, X}\left(t_{0}\right)+H_{d, \widetilde{R}}\left(t_{0}\right) \\
& \leq 1-H_{d, X}\left(t_{0}\right)+H_{d, R}\left(t_{0}\right)+m t_{0}^{n}=m t_{0}^{n}-\delta .
\end{aligned}
$$

We conclude that $m>\delta t_{0}^{-n}$, and therefore $m>a^{n}$ for some $a>1$, provided that $n$ is sufficiently large.

Combining our estimates, we obtain

$$
d_{H}\left(D_{n}\right) \geq \max \left(a^{n}, \log \left(D_{n}: D_{n+1}\right)\right) .
$$

Suppose first that $\alpha=\lim \sup \frac{\log \left(D_{n}: D_{n+1}\right)}{a^{n}}$ is finite. Then, for all $n$, there exists a constant $A$ such that

$$
\begin{aligned}
\log \left(H: D_{n}\right) & =\sum_{\nu=1}^{n-1} \log \left(D_{v}: D_{\nu+1}\right) \\
& \leq A+(\alpha+1) \sum_{\nu=1}^{n-1} a^{v} \leq A+\frac{\alpha+1}{a-1} a^{n}
\end{aligned}
$$


Since $D_{n}$ is a characteristic subgroup of $H$ and $d_{H}\left(D_{n}\right) \geq a^{n}$, we deduce our claim.

On the other hand, if $\lim \sup \frac{\log \left(D_{n}: D_{n+1}\right)}{a^{n}}$ is infinite, then we can pick a subsequence $\left(n_{i}\right)$ such that, for all $i$ and for all $m<n_{i}$, we have

$$
\frac{\log \left(D_{n_{i}}: D_{n_{i}+1}\right)}{a^{n_{i}}}>\frac{\log \left(D_{m}: D_{m+1}\right)}{a^{m}} .
$$

For such $n_{i}$, we have

$$
\begin{aligned}
\log \left(H: D_{n_{i}}\right) & =\sum_{\nu=1}^{n_{i}-1} \log \left(D_{v}: D_{\nu+1}\right) \\
& \leq \log \left(D_{n_{i}}: D_{n_{i}+1}\right) \sum_{\nu=1}^{n_{i}-1} a^{\nu-n_{i}} \\
& \leq \frac{1}{a-1} \log \left(D_{n_{i}}: D_{n_{i}+1}\right) \leq \frac{1}{a-1} d_{H}\left(D_{n_{i}}\right) .
\end{aligned}
$$

Again, our claim follows.

We can now prove Theorem 3.

Proof of Theorem 3. Let $G$ and $H$ be as in the theorem. By Proposition 7 (2), we may replace $H$ by an open normal subgroup, which is still generalized GolodShafarevich. Then Lemma 8 implies that $H$ satisfies the assumptions of Lemma 6 , and we conclude that $G$ has normal subgroup growth of type $n^{\log n}$.

\section{Groups with large meta-(elementary abelian) sections}

Let us prove part (1) of Theorem 2.

Proof of Theorem 2(1). Recall that $\Gamma$ is a $d$-generated group, $\Delta$ a normal subgroup of $\Gamma$ of finite index, $p$ a prime number, $H$ the pro- $p$ completion of $\Delta$, and $\Psi$ is the pre-image of $\Phi(H)=H^{p}[H, H]$, the Frattini subgroup of $H$, under the canonical map $\Delta \rightarrow H$. We write $L=\Phi_{H}(\Phi(H))=\Phi(H)^{p}[\Phi(H), H]$ for the normal Frattini subgroup of $\Phi(H)$ in $H$ and $\Lambda$ for its pre-image in $\Delta$.

Clearly, $\Psi$ and $\Lambda$ are characteristic subgroups in $\Delta$ and thus normal in $\Gamma$. Suppose that $x_{1}, \ldots, x_{d}$ are generators of $\Psi$ as a normal subgroup of $\Gamma$. Then $x_{1} \Lambda, \ldots, x_{d} \Lambda$ are generators of $\Psi / \Lambda$ as a normal subgroup of $\Gamma / \Lambda$. Hence, $X=\bigcup\left(x_{i} \Lambda\right)^{\Gamma / \Lambda}$ generates $\Psi / \Lambda$ as a subgroup. Since $\Psi / \Lambda$ is central in $\Delta / \Lambda$, we have $\Delta / \Lambda \leq C_{\Gamma / \Lambda}\left(x_{i} \Lambda\right)$, which implies

$$
\left|\left(x_{i} \Lambda\right)^{\Gamma / \Lambda}\right|=\left(\Gamma / \Lambda: C_{\Gamma / \Lambda}\left(x_{i} \Lambda\right)\right) \leq(\Gamma / \Lambda: \Delta / \Lambda)=(\Gamma: \Delta) .
$$


We deduce that

$$
d_{\Gamma}(\Psi) \geq d_{\Gamma / \Lambda}(\Psi / \Lambda) \geq \frac{d(\Psi / \Lambda)}{(\Gamma: \Delta)} .
$$

Notice that $H / L$ is the maximal CMEA-quotient of $H$. Because $\Psi / \Lambda$ is an elementary abelian $p$-group, we have

$$
\begin{aligned}
d_{\Gamma}(\Psi) & \geq \frac{d(\Psi / \Lambda)}{(\Gamma: \Delta)}=\frac{\log (\Psi: \Lambda)}{(\Gamma: \Delta)}=\frac{\log (\Phi(H): L)}{(\Gamma: \Delta)} \\
& =\frac{\log (H: L)-\log (H: \Phi(H))}{(\Gamma: \Delta)}=\frac{\mathrm{rk}_{c m} H-d(H)}{(\Gamma: \Delta)} \\
& \geq \frac{\mathrm{rk}_{c m} H-d(\Delta)}{(\Gamma: \Delta)} \geq \frac{\mathrm{rk}_{c m} H-d(\Gamma)(\Gamma: \Delta)}{(\Gamma: \Delta)}=\frac{\mathrm{rk}_{c m} H}{(\Gamma: \Delta)}-d,
\end{aligned}
$$

and the first part of Theorem 2 follows.

To prove part (2) of the theorem, we need the following lemma from Gerdau [5]. Since it has not been published, we include the proof here. Note that the statement is quite easy if $p \nmid|G|$, for, in that case, every module decomposes into a sum of simple modules, and there are only finitely many isomorphism types of simple modules of $\mathbb{F}_{p} G$.

Lemma 9. Let $G$ be a finite group and $p$ a prime number. Then there exists a constant $c>0$ depending only on $G$ and $p$ such that, for all $\mathbb{F}_{p} G$ modules $M$ of $\mathbb{F}_{p}$-dimension $d$, there exist submodules $M_{1} \leq M_{2} \leq M$ such that $M_{2} / M_{1}$ is the direct sum of at least $c d$ isomorphic simple modules.

Proof. For a module $M$, define $\ell(M)$ as the maximal length of an ascending series of submodules in a cyclic submodule of $M$. Since a cyclic $\mathbb{F}_{p} G$-module has dimension at most $|G|$, we have $\ell(M) \leq|G|$. We prove by induction on $\ell$, that there exists a constant $c_{\ell}>0$, such that for all modules $M$ with $\ell(M) \leq \ell$ there exists submodules $M_{1} \leq M_{2} \leq M$, such that $M_{2} / M_{1}$ is the direct sum of $c_{\ell} d$ isomorphic simple modules. We then take $c=c_{|G|}$.

Note that a simple module is cyclic, and therefore there are only finitely many isomorphism classes of simple modules. Define $N$ to be the submodule of $M$ generated by all simple submodules of $M$. Clearly $N$ is a direct sum of simple modules. Write $k_{1}=\sum \operatorname{dim}_{\mathbb{F}_{p}} S$ and $c_{1}=1 / k_{1}$, where the summation is over all isomorphism type of simple modules of $M$. Then $N$ contains a direct sum of at least $\operatorname{dim}_{\mathbb{F}_{p}} N / k_{1}=c_{1} \operatorname{dim}_{\mathbb{F}_{p}} N$ simple submodules of the same isomorphism class.

For $\ell=1$ any cyclic submodule of $M$ is simple. Thus, $N$ contains all the elements of $M$ and we have $N=M$. Let $M_{1}=1$ and $M_{2} \leq N$ be the direct sum of at least $c_{1} \operatorname{dim}_{\mathbb{F}_{p}} N=c_{1} d$ simple submodules of the same isomorphism class. 
We continue by induction on $\ell$. If $\operatorname{dim}_{\mathbb{F}_{p}} N>d / 2$, then $N$ contains a direct sum of $c_{1} \operatorname{dim}_{\mathbb{F}_{p}} N \geq c_{1} d / 2$ isomorphic simple modules. If $\operatorname{dim}_{\mathbb{F}_{p}} N \leq d / 2$, then $\ell(M / N) \leq \ell(M)-1$, as the first module in an ascending series of maximal length must be a simple module. By the induction hypothesis $\bar{M}=M / N$ contains submodules $\overline{M_{1}}<\overline{M_{2}}$, such that $\overline{M_{2}} / \overline{M_{1}}$ is the direct sum of at least $c_{\ell-1} d$ isomorphic simple modules. It is easy to deduce our claim with $c_{\ell}=\min \left(c_{1} / 2, c_{\ell-1}\right)$.

Just as in the case of vector spaces, direct sums of isomorphic modules have many submodules. The following is [10, Lemma 1].

Lemma 10. Let $G$ be a finite group, $M$ an $\mathbb{F}_{p} G$-module. Then there is some $c>0$ such that $M^{n}$ contains at least $e^{c n^{2}}$ submodules.

We are now ready to prove part (2) of Theorem 2.

Proof of Theorem 2(2). Let $\Delta, H$ and $\Delta_{i}$ be as in the theorem. Let $H_{i}$ be the closure of the image of $\Delta_{i}$ in $H$. Let $\Psi_{i}$ be the pre-image of $\Phi\left(H_{i}\right)$ in $\Delta$, and $\Lambda_{i}$ the pre-image of $\Phi_{H}(\Phi(H))$.

Let $\Omega$ be the kernel of the map $\Delta \rightarrow H$. Then $\Psi_{i}$ is generated by $\Omega,\left[\Delta, \Delta_{i}\right]$ and $\Delta_{i}^{p}$. Since all three groups are normal in $\Gamma, \Psi_{i}$ is also normal in $\Gamma$, and similarly $\Lambda_{i}$ is normal in $\Gamma$. Hence, $\Gamma$ acts on $\Psi_{i} / \Lambda_{i}$ by conjugation. Because $\Delta$ acts trivially on $\Psi_{i} / \Lambda_{i}$, we have that $\Psi_{i} / \Lambda_{i}$ is an $\mathbb{F}_{p}(\Gamma / \Delta)$-module. From Lemmas 9 and 10, it follows that there is some $c^{\prime}>0$, depending only on $\Gamma / \Delta$ and $p$, such that $\Psi_{i} / \Lambda_{i}$ has at least $e^{c^{\prime}\left(\operatorname{dim}_{\mathbb{F}} \Psi_{i} / \Lambda_{i}\right)^{2}}$ submodules. Since there is a bijection between submodules and normal subgroups of $\Gamma$, which are contained in the interval $\left(\Psi_{i}, \Lambda_{i}\right)$, we conclude that $\Gamma$ has at least $e^{c^{\prime}\left(\operatorname{dim}_{\mathbb{F}_{p}} \Psi_{i} / \Lambda_{i}\right)^{2}}$ normal subgroups of index at most $\left(\Gamma: \Lambda_{i}\right)$. Therefore, to prove the theorem, it suffices to show that there exists some $\delta>0$, independent of $i$, such that $\operatorname{dim}_{\mathbb{F}_{p}} \Psi_{i} / \Lambda_{i} \geq \delta \log \left(\Gamma: \Lambda_{i}\right)$.

As in the proof of part (1), we have

$$
\begin{aligned}
\log \left(\Phi\left(H_{i}\right): \Phi_{H_{i}}\left(\Phi\left(H_{i}\right)\right)\right) & =\operatorname{rk}_{c m} H_{i}-d\left(H_{i}\right) \\
& \geq \operatorname{rk}_{c m} H_{i}-d\left(\Delta_{i}\right) \\
& \geq \operatorname{rk}_{c m} H_{i}-d(\Gamma)\left(\Gamma: \Delta_{i}\right) .
\end{aligned}
$$

It follows from the assumption in the theorem that

$$
\begin{aligned}
\log \left(\Psi_{i}: \Lambda_{i}\right)=\log \left(\Phi\left(H_{i}\right): \Phi_{H}\left(\Phi\left(H_{i}\right)\right)\right) & \geq c\left(\Gamma: \Delta_{i}\right)^{2}-d(\Gamma)\left(\Gamma: \Delta_{i}\right) \\
& \geq c\left(\Gamma: \Delta_{i}\right)-d(\Gamma),
\end{aligned}
$$

so

$$
\left(\Gamma: \Delta_{i}\right) \leq \frac{\log \left(\Psi_{i}: \Lambda_{i}\right)+d(\Gamma)}{c} .
$$


We deduce that

$$
\begin{aligned}
\log \left(\Gamma: \Lambda_{i}\right) & =\log \left(\Gamma: \Delta_{i}\right)+\log \left(\Delta_{i}: \Psi_{i}\right)+\log \left(\Psi_{i}: \Lambda_{i}\right) \\
& \leq \log \left(\frac{\log \left(\Psi_{i}: \Lambda_{i}\right)+d(\Gamma)}{c}\right)+d\left(H_{i}\right)+\log \left(\Psi_{i}: \Lambda_{i}\right) \\
& \leq \log \left(\Psi_{i}: \Lambda_{i}\right)+d(H)\left(H: H_{i}\right)+\log \left(\Psi_{i}: \Lambda_{i}\right) \\
& \leq d(H)\left(\Gamma: \Delta_{i}\right)+2 \log \left(\Psi_{i}: \Lambda_{i}\right) \\
& \leq \frac{d(H)}{c} \log \left(\Psi_{i}: \Lambda_{i}\right)+\frac{d(H) d(\Gamma)}{c}+2 \log \left(\Psi_{i}: \Lambda_{i}\right) \\
& \leq\left(\frac{d(H)}{c}+3\right) \log \left(\Psi_{i}: \Lambda_{i}\right),
\end{aligned}
$$

provided that $\left(\Psi_{i}: \Lambda_{i}\right)$ is sufficiently large. Putting $\delta=\left(\frac{d(H)}{c}+3\right)^{-1}$, we have $\operatorname{dim}_{\mathbb{F}_{p}} \Psi_{i} / \Lambda_{i}=\log \left(\Psi_{i}: \Lambda_{i}\right) \geq \delta \log \left(\Gamma: \Lambda_{i}\right)$ as required, and Theorem 2(1) is proven.

Proof of Proposition 4. Let $\widehat{F}_{d}$ be the free pro- $p$ group of rank $d$. Clearly, the normal growth of $\widehat{F_{d}} / \gamma_{2}\left(\widehat{F}_{d}^{\prime}\right)$ is a lower bound for the normal growth of $F_{d} / \gamma_{2}\left(F_{d}^{\prime}\right)$. Let $N$ be the normal subgroup of $\widehat{F}_{d}$ such that $\widehat{F}_{d} / N \cong\left(\mathbb{Z} / p^{k} \mathbb{Z}\right)^{d}$. Then we have $N \geq \widehat{F}_{d}^{\prime}$, and therefore $\Phi(N) \geq \widehat{F}_{d}^{\prime \prime}=\gamma_{1}\left(\widehat{F}_{d}^{\prime}\right)$ and $\Phi_{N}(\Phi(N)) \geq \gamma_{2}\left(\widehat{F}_{d}^{\prime}\right)$. Thus, $\left(\widehat{F}_{d}: N\right),(N: \Phi(N))$ and $\Phi(N) / \Phi_{N}(\Phi(N))$ do not change when we factor by $\gamma_{2}\left(\widehat{F}_{d}^{\prime}\right)$. Let $d_{1}=(d-1)\left(\widehat{F}_{d}: N\right)+1$; then $N \cong \widehat{F}_{d_{1}}$. Since $N$ is a free pro- $p$ group, it surjects onto the free CMEA group with $d_{1}$ generators. The latter has order

$$
p^{d_{1}+\left(\begin{array}{c}
d_{1}+1 \\
2
\end{array}\right)}>p^{d_{1}^{2} / 2}, \quad \text { so } \quad \operatorname{rk}_{c m} N>\frac{d_{1}^{2}}{2} .
$$

Hence,

$$
\operatorname{rk}_{c m} N>\frac{d_{1}^{2}}{2}=\frac{1}{2}\left((d-1)\left(\widehat{F}_{d}: N\right)+1\right)^{2}>\frac{(d-1)^{2}}{2}\left(\widehat{F}_{d}: N\right)^{2},
$$

and we can apply Theorem 2 . On the other hand, $F / \gamma_{2}\left(F_{d}^{\prime}\right)$ is not Golod-Shafarevich since, as Zelmanov showed in [15], Golod-Shafarevich groups always contain non-abelian free pro- $p$ subgroups, which $F / \gamma_{2}\left(F_{d}^{\prime}\right)$ clearly does not.

Proof of Corollary 1. Let $\Gamma$ be as in the corollary. Since the center of $\Gamma$ is trivial, we can view $\Gamma$ as a subgroup of $\operatorname{Aut}(\Gamma)$. A subgroup of $\Gamma$ is characteristic in $\Gamma$ if and only if it is normal in $\operatorname{Aut}(\Gamma)$. Since $(\operatorname{Aut}(\Gamma): \Gamma)$ is finite, we can apply Theorem 2 to the pair $\operatorname{Aut}(\Gamma)>\Gamma$ in place of $\Gamma>\Delta$ and find that $\Gamma$ has characteristic subgroup growth of type $n^{\log n}$.

It remains to show that free products of finite groups have trivial center and finite outer automorphism group. The statement about the center is easy. In fact, as 
non-abelian free groups have trivial center, every element of the center of $A * B$ is contained in a conjugate of $A$ or $B$. But elements in conjugates of $A$ do not commute with elements in conjugates of $B$; hence the center is trivial. The finiteness of the outer automorphism group was shown by Pettet [11, Proposition 2.5].

\section{Problems and remarks}

Lemma 6 falls short of our expectations in two aspects. First, it is unfortunate that the subgroups $N$ are required to be characteristic subgroups of $H$ rather than normal. We therefore ask the following.

Problem 3. Does there exist a pro-p group $G$ such that, for some $c>0$, there exist infinitely many open normal subgroups $N$ with $d_{G}(N) \geq c \log (G: N)$, but $\lim \frac{d_{G}(C)}{\log (G: C)}=0$, as $C$ ranges over characteristic open subgroups?

The second problem is that Lemma 6 only gives information on normal growth of type $n^{\log n}$ because our counting methods are very crude. To give an upper bound for the number of normal subgroups, one considers all chains of the form $G>N_{1}>\cdots>N_{n}$, disregarding the facts that $N_{n}$ might be contained in many different chains and that not all normal subgroups need the maximal number of generators. For the lower bound, one considers one normal subgroup $N$ which needs many generators and counts only normal subgroups between $N$ and $\Phi_{G}(N)$. Both estimates appear heavily wasteful, but, for growth types $n^{\log n}$ and larger, the difference is actually quite small. However, if

$$
\max _{(G: N)=n} d_{G}(N) \approx f(\log n)
$$

with $f(k)=o(k)$, one only gets a lower bound of type $e^{f(\log n)^{2}}$ and an upper bound $e^{f(\log n) \log n}$. In particular, we do not know whether an analogue of Lemma 5 exists. We therefore ask the following.

Problem 4. Do there exist pro-p groups $G, H$ such that, for all sufficiently large $k$, we have

$$
\max _{(G: N) \leq p^{k}} d_{G}(N)>\max _{(H: K) \leq p^{k}} d_{H}(K),
$$

but

$$
\frac{\log s_{p^{k}}^{\triangleleft}(G)}{\log s_{p^{k}}^{\triangleleft}(H)} \underset{k \rightarrow \infty}{\longrightarrow} 0 ?
$$

In addition, we believe that some generalization of Theorem 2 should be true, although we are not sure what form it should take. Informally, Theorem 2 assumes that $G$ contains infinitely many normal subgroups, which map onto CMEA groups of "size comparable to the corresponding quantity in a free group". If we replace 
"CMEA" by "elementary abelian" in this statement, we obtain positive upper rank gradient which is equivalent to exponential subgroup growth. Here we define $\mathrm{rg}^{+}$ and $\mathrm{rg}^{-}$the upper and the lower rank gradient of a pro- $p$ group $G$ respectively as

$$
\begin{aligned}
& \mathrm{rg}^{+}=\sup _{G>U_{1}>U_{2}>\ldots} \lim _{n \rightarrow \infty} \frac{d\left(U_{i}\right)}{\left(G: U_{i}\right)}, \\
& \mathrm{rg}^{-}=\inf _{G>U_{1}>U_{2}>\ldots} \lim _{n \rightarrow \infty} \frac{d\left(U_{i}\right)}{\left(G: U_{i}\right)},
\end{aligned}
$$

where infimum and supremum are taken over all descending chains of finite index subgroups.

However, positive upper rank gradient does not imply large normal growth, for example, $G$, the pro- $p$ completion of the restricted wreath product $\Gamma=\mathbb{Z} \imath \mathbb{F}_{p}$, has polynomial normal growth as shown in [7, example after Theorem 9.2]; however, let us see that it has positive upper rank gradient.

Let $\Sigma$ be the base group of $\Gamma$. We have a map $\psi_{k}: \Gamma \rightarrow P_{k}=C_{p^{k}} \imath \mathbb{F}_{p}$. Let $B$ be the base group of $P_{k}$, and let $\Delta$ be the pre-image of $B$ and $N$ its closure in $G$. Then $\Delta$ is a normal subgroup of $\Gamma$ of index $p^{k}, \Sigma \leq \Delta$, and $[\Delta, \Delta] \Delta^{p}$ is in the kernel of $\psi_{k}$ because $B$ is an elementary abelian $p$-group. Hence,

$$
\begin{aligned}
(N: \Phi(N)) & \geq\left(\Delta:[\Delta, \Delta] \Delta^{p}\right) \geq\left(\Delta \cap \Sigma:[\Delta, \Delta] \Delta^{p} \cap \Sigma\right) \\
& \geq\left(\Sigma: \operatorname{ker} \psi_{k} \cap \Sigma\right)=|B|=p^{p^{k}},
\end{aligned}
$$

and we obtain $d(N) \geq p^{k}=[\Gamma: \Delta]=[G: N]$. In particular, the upper rank gradient of $G$ is as large as the rank gradient of a free group with 2 generators, and the subgroup growth of $G$ is exponential.

One reason that explains the discrepancy between the large subgroup growth and the moderate normal growth is the fact that if a subgroup $U$ requires many generators, then it contains essentially the whole base group, while $\Phi(U)$ cuts deeply into the base group and needs only few generators. In particular, there are no large CMEA sections in $G$ because most subgroups need only few generators. It would be interesting to know what happens if we circumvent this obstacle by assuming that all subgroups need many generators. We therefore ask the following problem.

Problem 5. If $G$ has positive lower rank gradient, does $G$ necessarily have large normal growth?

It is not hard to see that the conditions in Theorem 2 imply exponential subgroup growth. Thus, comparing it to Theorem 3 naturally leads to the following problem.

Problem 6. Can one give an example of a generalized Golod-Shafarevich group with subexponential subgroup growth? If not, can one give some other example of 
a generalized Golod-Shafarevich group to which Theorem 2 cannot be applied? More generally, does the assumption that $G$ has normal subgroup growth of type $n^{\log n}$ imply any other largeness properties?

The last question is probably very difficult, as, for example, the pro- 2 completion of Grigorchuk's group or the Nottingham group contain non-abelian free pro- $p$ subgroups, but have only a bounded number of normal subgroups of any given index. Finally, we would like to know the answer to the original claim from [10].

Problem 7. Let $\Gamma$ be a large group, that is, it contains a finite index subgroup $\Delta$, which projects onto a non-abelian free group $F$. Does $\Gamma$ have normal subgroup growth of type $n^{\log n}$ ?

Note that a negative answer to Problem 2 would immediately resolve this question in the positive.

For characteristic subgroup growth, the first question one might consider is the following.

Problem 8. What is the characteristic subgroup growth of a non-abelian free group?

This question was posed by I. Rivin on MathOverflow (see [17]) and seems intriguingly difficult. W. Thurston gave an argument that the growth should be similar to the characteristic subgroup growth of $\mathbb{Z}^{n}$. However, the direct approach leads to well-known open problems on the product replacement algorithm. A first step in this direction could be the following problem, which is due to Lubotzky.

Problem 9. Is there a characteristic subgroup $C$ in $F_{3}$ such that $F_{3} / C$ is a finite simple group?

Acknowledgments. We would like to thank $\mathrm{H}$. Wilton for suggesting the example in Corollary 1 of the free product of two non-trivial finite groups and L. Mosher for pointing us to the literature about its outer automorphisms; see the question in MathOverflow [16].

\section{Bibliography}

[1] J. O. Button and A. Thillaisundaram, Applications of $p$-deficiency and $p$-largeness, Int. J. Algebra Comput. 21 (2011), 547-574.

[2] M. Ershov, Kazhdan quotients of Golod-Shafarevich groups, with appendices by A. Jaikin-Zapirain, Proc. Lond. Math. Soc. (2) 102 (2011), 599-636. 
[3] M. Ershov, Golod-Shafarevich groups: A survey, Int. J. Algebra Comput. 22 (2012), Article ID 1230001.

[4] M. Ershov and A. Jaikin-Zapirain, Groups of positive weighted deficiency and their application, J. reine angew. Math. 677 (2013), 71-134.

[5] V. Gerdau, Untergruppenwachstum virtuell freier pro-p Gruppen, Diplomarbeit, Freiburg, 2010.

[6] A. Lubotzky, Enumerating boundedly generated finite groups, J. Algebra 238 (2001), 194-199.

[7] A. Lubotzky and D. Segal, Subgroup Growth, Progr. Math. 212, Birkhäuser, Basel, 2003.

[8] W. Magnus, Beziehungen zwischen Gruppen und Idealen in einem speziellen Ring, Math. Ann. 111 (1935), 259-280.

[9] A. Mann, Enumerating finite groups and their defining relations, J. Group Theory 1 (1998), 59-64.

[10] T. W. Müller and J.-C. Puchta, Normal growth of large groups II, Arch. Math. 84 (2005), 289-291.

[11] M. R. Pettet, Virtually free groups with finitely many outer automorphisms, Trans. Amer. Math. Soc. 349 (1997), no. 11, 4565-4587.

[12] D. Segal, On the growth of ideals and submodules, J. Lond. Math. Soc. (2) 56 (1997), 245-263.

[13] J.-C. Schlage-Puchta, A p-group with positive rank gradient, J. Group Theory 15 (2012), 261-270.

[14] J.-C. Schlage-Puchta and J. Wolfart, How many quasiplatonic curves?, Archiv Math. 86 (2006), 129-132.

[15] E. Zelmanov, On groups satisfying the Golod-Shafarevich condition, in: New Horizons in pro-p groups, Progr. Math. 184, Birkhäuser, Boston (2000), 223-232.

[16] http://mathoverflow.net/questions/265055/

big-groups-g-with-trivial-outg.

[17] http://mathoverflow.net/questions/51053/ counting-characteristic-subgroups.

Received March 24, 2017; revised August 6, 2019.

\section{Author information}

Yiftach Barnea, Department of Mathematics, Royal Holloway,

University of London, Egham, Surrey, TW20 0EX, United Kingdom.

E-mail: y.barnea@rhul.ac.uk

Jan-Christoph Schlage-Puchta, Institut für Mathematik,

Ulmenstr. 69, 18051 Rostock, Germany.

E-mail: jan-christoph.schlage-puchta@uni-rostock.de 